\title{
AUXIN RESPONSE FACTOR 1 Acts as a Positive Regulator in the Response of Poplar to Trichoderma asperellum Inoculation in Overexpressing Plants
}

\author{
Yue-Feng Wang ${ }^{1,2,3,+} \oplus^{\oplus}$, Xue-Yue Hou ${ }^{1,+}{ }^{,}$Jun-Jie Deng ${ }^{1}$, Zhi-Hong Yao ${ }^{1}$, Man-Man Lyu ${ }^{1}$ and \\ Rong-Shu Zhang $1, * \mathbb{B}$ \\ 1 College of Landscape Architecture, Northeast Forestry University, 26 Hexing Road, Harbin 150040, China; \\ wangyf777@ibcas.ac.cn (Y.-F.W.); houxueyue@nefu.edu.cn (X.-Y.H.); fireflyndmoon@nefu.edu.cn (J.-J.D.); \\ dblydx2015@nefu.edu.com (Z.-H.Y.); lvmanman@nefu.edu.cn (M.-M.L.) \\ 2 Photosynthesis Research Center, CAS Key Laboratory of Photobiology, Institute of Botany, Chinese Academy \\ of Sciences, Beijing 100093, China \\ 3 College of Life Sciences, Graduate University of Chinese Academy of Sciences, Beijing 100049, China \\ * Correspondence: zrs6504@nefu.edu.cn; Tel.: +86-0451-8219-0610 \\ + Equal contribution.
}

Received: 14 January 2020; Accepted: 15 February 2020; Published: 19 February 2020

check for updates

\begin{abstract}
Numerous Trichoderma strains have been reported to be optimal biofertilizers and biocontrol agents with low production costs and environmentally friendly properties. Trichoderma spp. promote the growth and immunity of plants by multiple means. Interfering with the hormonal homeostasis in plants is the most critical strategy. However, the mechanisms underlying plants' responses to Trichoderma remain to be further elucidated. Auxin is the most important phytohormone that regulates almost every aspect of a plant's life, especially the trade-off between growth and defense. The AUXIN RESPONSE FACTOR (ARF) family proteins are key players in auxin signaling. We studied the responses and functions of the PAPapARF1 gene in a hybrid poplar during its interaction with beneficial T. asperellum strains using transformed poplar plants with PdPapARF1 overexpression (on transcription level in this study). We report that PdPapARF1 is a positive regulator for promoting poplar growth and defense responses, as does T. asperellum inoculation. PdPapARF1 also turned out to be a positive stimulator of adventitious root formation. Particularly, the overexpression of PdPapARF1 induced a $32.3 \%$ increase in the height of 40 -day-old poplar plants and a $258 \%$ increase in the amount of adventitious root of 3-week-old subcultured plant clones. Overexpressed PdPapARF1 exerted its beneficial functions through modulating the hormone levels of indole acetic acid (IAA), jasmonic acid (JA), and salicylic acid (SA) in plants and activating their signaling pathways, creating similar results as inoculated with T. asperellum. Particularly, in the overexpressing poplar plants, the IAA level increased by approximately twice of the wild-type plants; and the signaling pathways of IAA, JA, and SA were drastically activated than the wild-type plants under pathogen attacks. Our report presents the potential of ARFs as the crucial and positive responders in plants to Trichoderma inducing.
\end{abstract}

Keywords: poplar; ARF1; Trichoderma; growth-promotion; hormone levels; hormone signaling pathways

\section{Introduction}

With the increasing population and environmental problems, sustainable agriculture and forestry are now in great demand. Biofertilizers and biocontrol agents who have low production costs and 
environmental impacts are optimal solutions for meeting such needs. Especially, Trichoderma spp. have received much attention for their functions as mycofungicides and plant growth promoters [1]. Trichoderma spp. usually exist in the rhizosphere, while some isolates can act as endosymbionts of plants [2]. The beneficial effects of Trichoderma are the overall outcomes of the interactions between Trichoderma, soil ecosystems, and plants. Accordingly, the mechanisms underlying the benefits of Trichoderma have been explored and uncovered from multiple perspectives [1,3-6]. One important growth-promoting mechanism is modifying the levels of phytohormones, including ethylene, cytokinin, auxin, or their related compounds in plant rhizosphere and root $[3,6]$. Some Trichoderma species were reported to produce gibberellin-related molecules (GAs) or zeatin [7]. Some can regulate the plant ethylene level by modifying the concentration of its immediate precursor, 1-aminocyclopropane-1-carboxylate (ACC) by ACC deaminase [8]. More reports demonstrated that some Trichoderma species could produce or degrade in vitro indole acetic acid (IAA), namely auxin, to create optimal IAA concentrations for plant growth [9-11].

Phytohormones regulate plant growth, development, as well as immunity and resistance against stresses through an interconnected network formed by signaling pathways [12]. Salicylic acid (SA), jasmonic acid (JA), and ethylene (ET) are crucial regulators of plant defense and resistance. Their signaling cascades cross paths with GA and IAA through hub proteins such as DELLA and EIN3 [13,14]. The biocontrol mechanisms of Trichoderma are highly diverse, which in turn makes Trichoderma spp. ubiquitously applicable agents [6]. One underlying mechanism is that Trichoderma activates the signaling or metabolism of SA and/or JA in plants, thus inducing systemic resistance (ISR), occasionally accompanied by systemic acquired resistance (SAR) [7,15]. Multiple reports have confirmed that Trichoderma inoculation can increase the levels of SA and JA, trigger ISR by SA-dependent manner while also involving JA/ET signaling pathways [16-18].

Under natural conditions, plants are constantly balancing between growth and defense [19]. Auxin is the key regulator of plant morphogenesis and growth [14]. In recent years, its roles as the nexus in plant-microbe interactions have emerged [20]. Auxin homeostasis in the plant is modified by concerted auxin biosynthesis, conjugation, and transport. Auxin signal transduction is achieved through binding to TRANSPORT INHIBITOR RESPONSE 1 (TIR1) and AUXIN SIGNALING F-BOX (AFB) receptors in the nucleus, which subsequently induces the proteolysis of AUXIN/INDOLE-3-ACETIC ACID (AUX/IAA) repressors and depress AUXIN RESPONSE FACTORs (ARFs) to activate the transcription of downstream auxin-responsive genes [14]. The ARF family proteins play a key role in auxin signaling and confer specificity to downstream responsive genes [21]. To date, the growth-promoting effects of Trichoderma spp. involving auxin signals have been attributed to the production of auxin-related compounds in vitro [9-11]. However, the internal responsive mechanisms in plants have not been elucidated. With such backgrounds, we studied the role of ARF1 in the interaction between a hybrid poplar Populus davidiana $\times$ P. alba var. pyramidalis (PdPap) and a beneficial T. asperellum strain.

\section{Results}

\subsection{PdPapARF1 Expression Is Responsive to T. asperellum Inoculation}

The DNA sequence and coding sequence (cds) of PdPapARF1 were cloned and submitted to GenBank (with Accession No. KP165071 and KM113035.1, respectively). The coding sequence of PdPapARF1 had $91.03 \%, 98.23 \%$, and $77.80 \%$ similarities with its orthologs in P. trichocarpa, Potri.004G228800.2, Potri.003G001000.1, and AtARF1 (AT1G59750.1) of Arabidopsis thaliana, respectively. In previous studies, we found that the T. asperellum strains ACCC32492 (Ta492) and ACCC30536 (Ta536) were both beneficial for poplar with Ta536 demonstrating the best effects among the three individual T. asperellum strains and that inoculation with mixed Trichoderma strains had even better beneficial effects [22,23]. So, we examined the expression of PdPapARF1 in response to Ta536 or Ta492 or the combination of four T. asperellum strains (Ta536+Ta492+T. asperellum ACCC $31650+T$. asperellum T4) by quantitative real-time polymerase chain reaction (qRT-PCR). Under field conditions, PdPapARF1 
expression in the leaves and roots of one-month-old poplar plants were rapidly induced by each or the combination of T. asperellum strains as early as $0.5 \mathrm{~h}$ after inoculation (HAI). Mixed inoculation resulted in the highest expression within an early response period of 2 HAI (Figure 1).
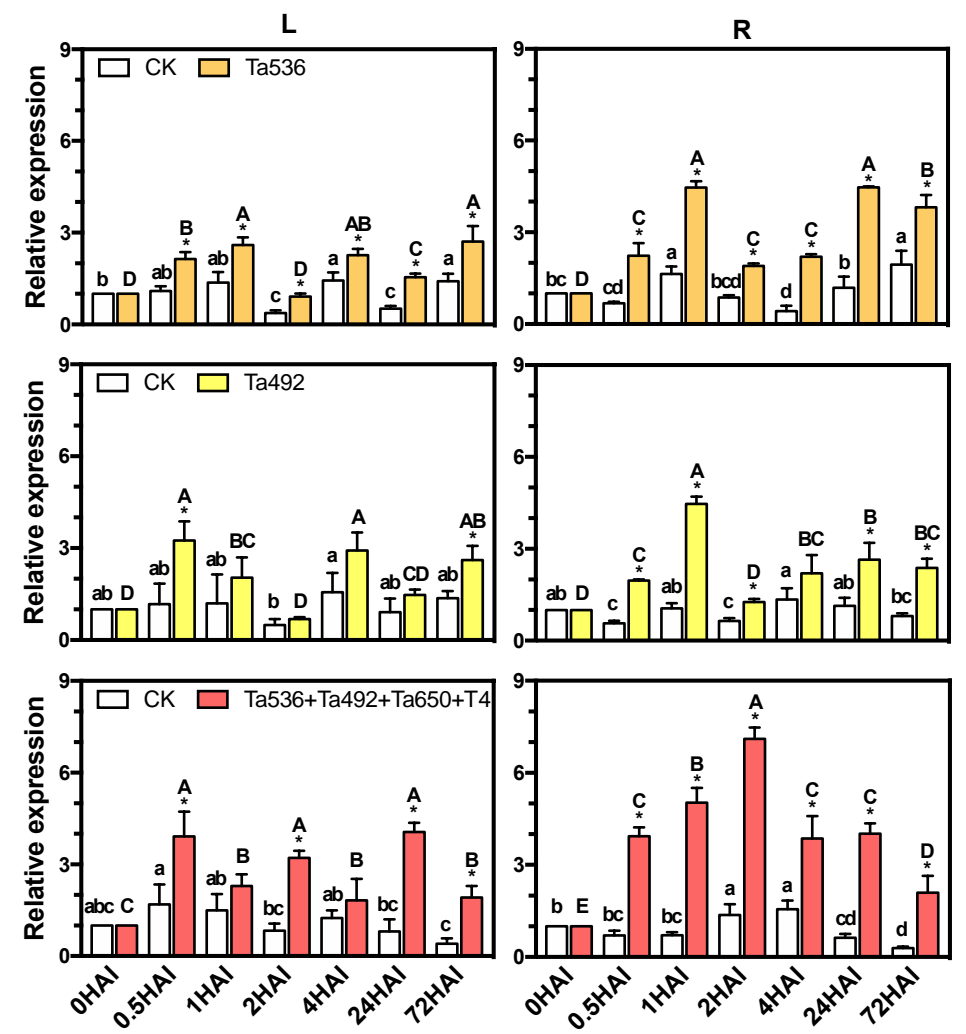

Figure 1. The expression of PdPapARF1 in response to the inoculation of different beneficial T. asperellum strains determined by q-RT-PCR. L, mature leaf. R, root. HAI, hour(s) after inoculation. Ta650, T. asperellum ACCC31650. T4, T. asperellum T4. Different capital letters represent significant differences among the inoculated plant samples taken at different times (HAI); different lowercase letters represent significant differences among the CK plant samples taken at different times; * significant difference between inoculated and CK plants at the same time. All significances were at $p<0.05$. All experiments were performed with three biological replicates with each replicate pooled of 10 plants and three technical replicates.

\subsection{Production of Transgenic Poplar with Modified Expression of PdPapARF1}

To confirm that PAPAPARF1 acted as a positive regulator in the poplars inoculated with Trichoderma, wild type (WT) PdPap plants were transformed with constructed vectors to generate plants overexpressing PdPapARF1 or with down-regulated expression of PdPapARF1. After screening by the expression of PdPapARF1 through qRT-PCR, four overexpression lines (named PdPapARF1 OX1-4) and four RNAi lines (named PAPapARF1 RNAi1-4) were obtained (Figure 2a). The growth traits of these transgenic poplar plants were subsequently observed. 


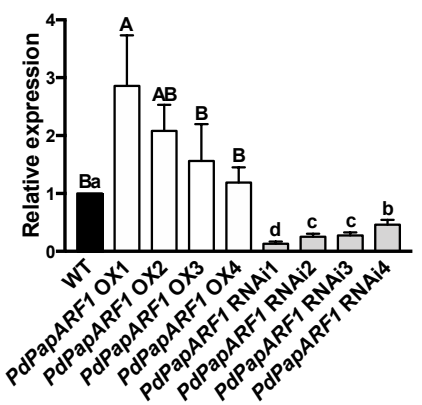

(a)
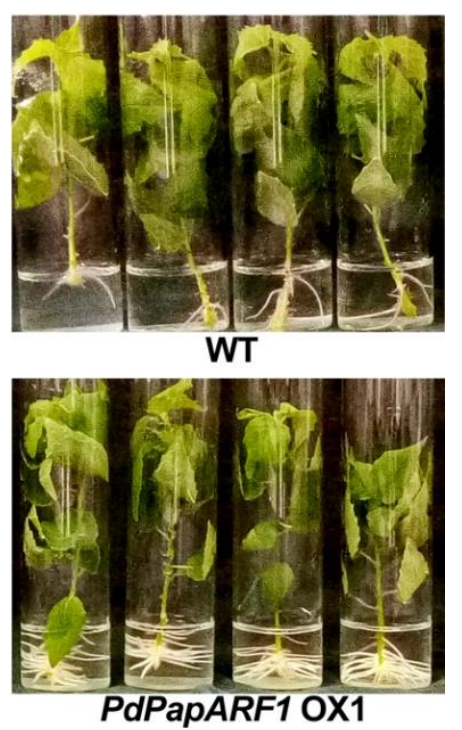

(c)
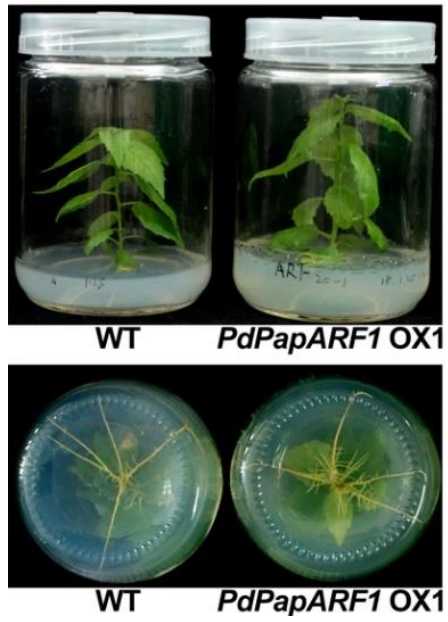

(b)
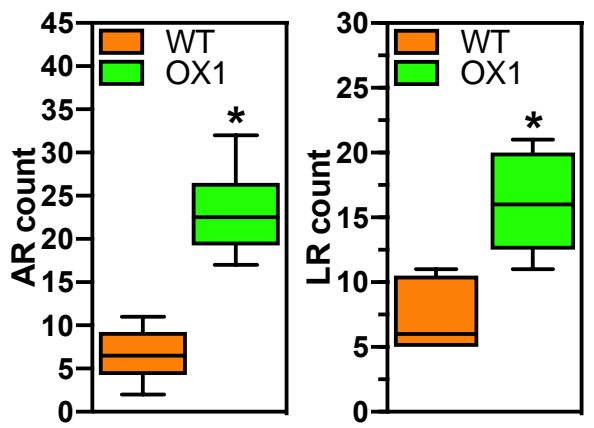

(e)

Figure 2. Cont. 


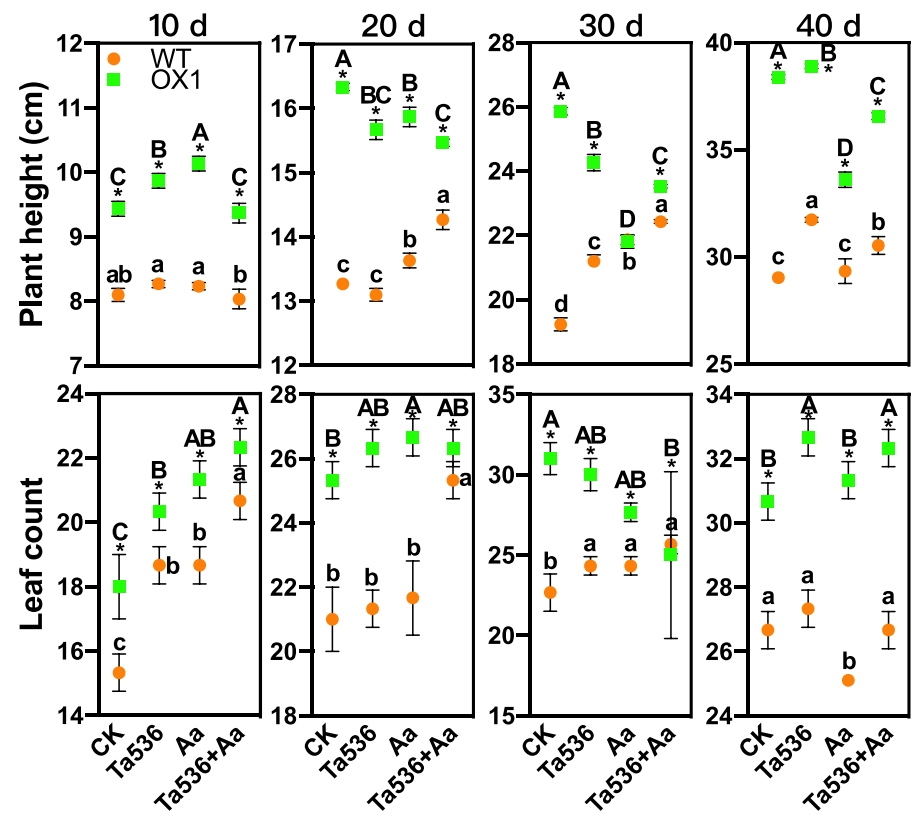

(d)

Figure 2. The identification, phenotypes, and growth traits of transformed poplar plants. (a) The relative expression of PdPapARF1 in the transgenic plant lines determined by qRT-PCR. Data were achieved from three technical replicates. (b) Three-week-old subcultured poplar plants in $8 \mathrm{~cm}$ (bottom diameter) vessels. The two upper panels show the plants and the two lower panels show their roots. (c) Three-week-old poplar plants in $3.5 \mathrm{~cm}$ (bottom diameter) vessels showing an evident increase of adventitious roots of the OX1 plants. (d) Growth dynamics of the WT and OX1 plants undergone different 48-h-treatments (see methods for details), then grew in pot soil for 40 days. d, day(s). Data on 0 days did not have any significant difference between the groups; thus, those data are not shown. For each group and each treatment, $n=3$. (e) The total amount of adventitious roots (AR) per plant (AR count, $n=8$ ) and the total amount of lateral root (LR) per AR (LR count, $n=4$ ). Different capital letters represent significant differences among the OX plants treated with different inoculations; different lowercase letters represent significant differences among the WT plants treated with different inoculations; * significant difference between OX and WT plants undergone the same inoculation. All significances were at $p<0.05$.

\subsection{PdPapARF1 Overexpression Promoted Growth and Adventitious Root Formation}

Within the sterile culturing stage, transformed poplar plants with different PdPapARF1 expression levels already demonstrated an evident difference in growth. The PdPApARF1 OX plants showed more rapid shoot growth, increased number of adventitious roots and lateral roots compared with WT. The PdPapARF1 RNAi plants showed severe tardiness in shoot growth, leaf size, and the number of adventitious roots (Figure 2b,c, Supplementary Figure S1). In this study, our focus is on the positive effects brought by Trichoderma inoculation. So far, our results suggest that PdPapARF1 is a positive responder toward Trichoderma inducing, and a positive regulator of poplar growth, as is Trichoderma. Hence, we conducted further studies only on the OX plants, using the PdPapARF1 OX1 (referred to as OX1 in the text hereafter) line, which had the highest expression of PdPapARF1.

Infection with pathogen fungi may impair the growth of host plants besides causing disease symptoms. Trichoderma, however, is a biocontrol agent that is supposed to balance out the negative effects brought by pathogens to an extent. Hence, we treated the subcultured clones of OX1 and WT plants with sole inoculation of Ta536 on the root, or pathogenic Alternaria alternata (Aa) inoculation on the leaves, or duel inoculations of Ta536+Aa for $48 \mathrm{~h}$, then grew the plants in soil for 40 days to monitor their growths. It takes up to thirty days for the subcultured PdPap plants to gradually adapt to the soil. Promoted shoot and leaf growths compared with WT were observed in the OX1 plants 
under each treatment at all measured time points. After 20 days, the Ta536 inoculation on WT began to show significant $(p<0.05)$ improving effects on the shoot growth (plant height). On the contrary, no promoting effects of Ta536 on the OX1 plants were observed before Day 40 (Figure 2d, Supplemental Table S1), suggesting that the overexpression of PdPapARF1 alone was competent to promote poplar growth, namely serving the purpose of Ta536, without any extra inoculation. Our results demonstrate that PAPapARF1 is a regulator that confers the growth-promoting benefits of T. asperellum inoculation. Extra Ta536 inoculation on the OX1 plants could not further improve poplar growth, suggesting other unknown mechanisms limiting the growth of poplar, possibly for maintaining the balance between vegetative growth and other life events of the plant.

\subsection{PdPapARF1 Overexpression Altered the Response of Poplar Leaf to A. alternata Infection}

Oxidative burst, which constitutes the production of reactive oxygen species (ROS) including superoxide and $\mathrm{H}_{2} \mathrm{O}_{2}$ at the attempted site of infection, is a rapid defend reaction of plants toward pathogen attack. ROS are essential signaling molecules when plants are under stress. However, the accumulation of ROS causes damage to plant tissues [24]. This reaction in resistant cultivars is more rapid and evident than susceptible cultivars [25]. We performed 3,3'-diaminobenzidine (DAB) staining assays to examine the reaction of OX1 plants when infected by Aa. The results reflected a much stronger oxidative burst in the OX1 plants compared with WT, indicating potentially stronger disease resistance to A. alternata. Furthermore, in the infected plants, the uninoculated leaves also displayed an accumulation of ROS to an extent, which was not observed in the OX1 plants (Figure 3). These results reflected ROX accumulation on a systemic scale in WT plants when subjected to biotic stress, while in the OX1 plants, the ROS accumulation was locally controlled at the infection site, giving proper protection to the uninfected plant compartments.

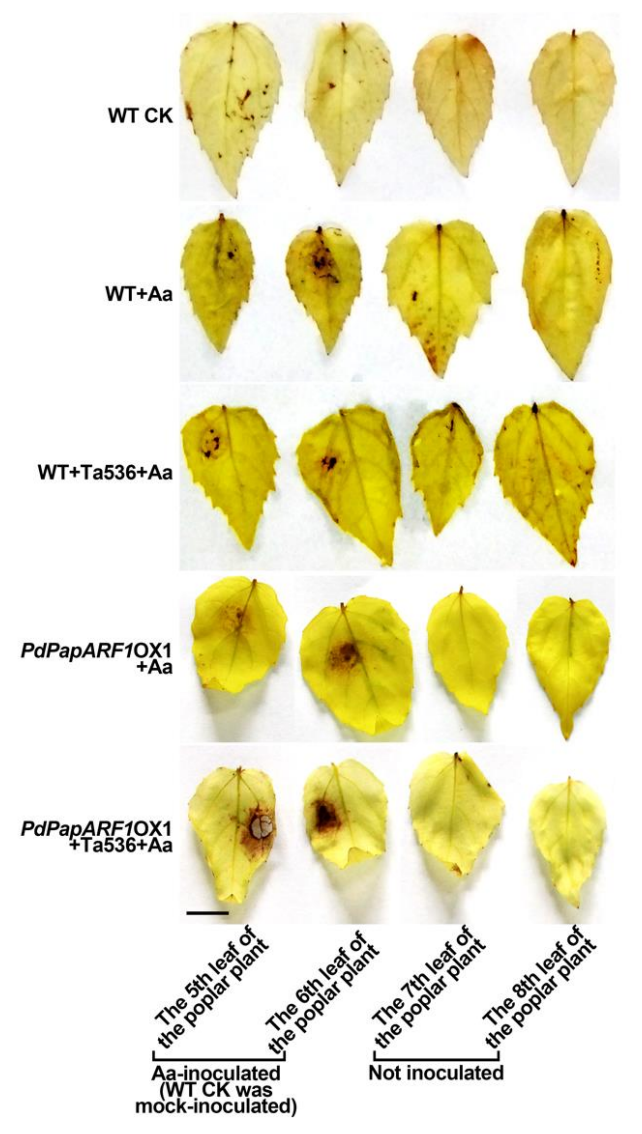

Figure 3. The 3,3'-diaminobenzidine (DAB) staining results of the leaves of three-week-old poplar clones subcultured under sterile conditions. $B a r=1 \mathrm{~cm}$. Please note that all the inoculated leaves were punctured once, including in mock-inoculation. See details in the material and methods section. 


\subsection{PdPapARF1 Overexpression Regulated Hormone Levels in Planta}

We examined the levels of IAA, JA, and SA in the young tissues (shoot tip, ST), mature leaves (L), and roots (R) of WT and OX1 plants undergone sole Ta536 or Aa, or the duel Ta536+Aa inoculations. The overexpression of PdPapARF1 resulted in extensive changes in the levels of these three phytohormones differentially in the three examined plant compartments. Notably, compared to WT, substantial increases of IAA, JA levels in the shoot tip, and JA, SA levels in the root were demonstrated (Figure 4, Supplemental Table S2). These findings suggest that PdPapARF1 is a factor for regulating the levels of major growth- and defense-related hormones during poplar's response to Trichoderma.

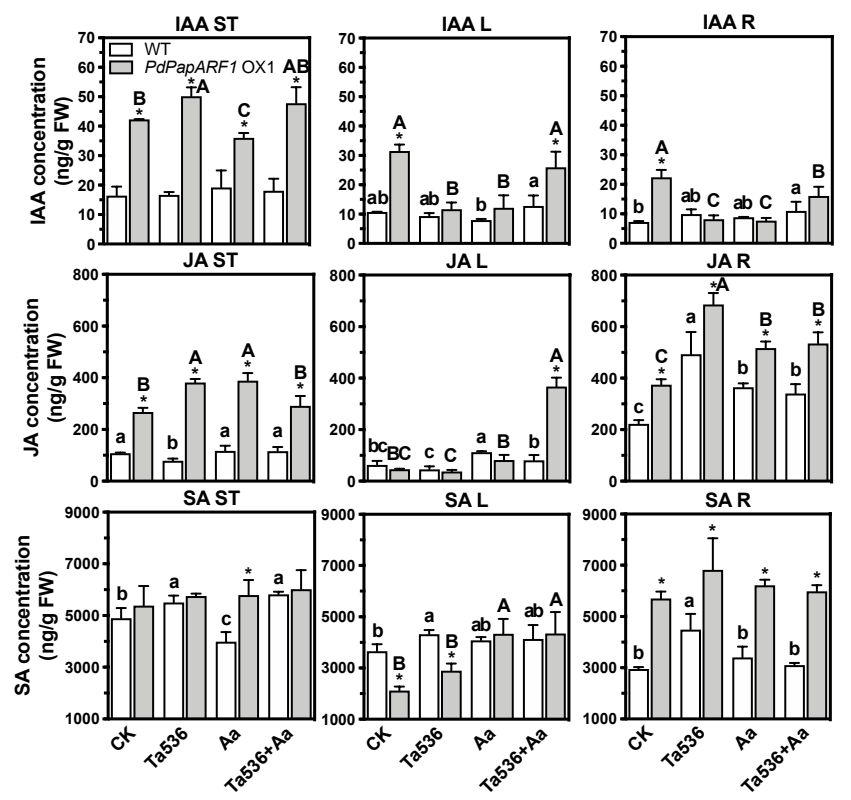

Figure 4. The concentrations of indole acetic acid (IAA), jasmonic acid (JA), and salicylic acid (SA). The hormone concentrations of three poplar compartments, the shoot tip (ST), inoculated leaves (L), and root (R) under no treatment (CK) or fungal treatments (Ta536, Aa, or Ta536+Aa) are shown. FW, fresh weight. Different capital letters represent significant differences among the OX plants treated with different inoculations; different lowercase letters represent significant differences among the WT plants treated with different inoculations; * significant difference between OX and WT plants undergone the same inoculation. Insignificant differences are not marked. All significances were at $p<0.05$. All experiments were performed with three biological replicates with each replicate pooled of three plants. The replicates of OX plants were the clones of the line OX1.

\subsection{PdPapARF1 Overexpression Influenced the IAA, JA and SA Signaling Cascades in Planta}

With the above results, subsequently, we explored whether the signaling pathways of these hormones were also influenced by PdPapARF1 overexpression. The expression of genes corresponding to the key elements of the JA signaling cascade, COI1, JAZ5, MYC2, and those of the SA signaling cascade, NPR1, TGA, PR1 were determined by qRT-PCR. Gene expression was separately investigated in three poplar compartments, namely ST, L, and R of WT, and OX1 plants undergone Ta536, Aa, or Ta536+Aa inoculations. The results demonstrated pronounced and differential changes in the expression levels of most of these genes between untreated WT and OX1 plants. When subjected to the fungal inoculation, the expression of more genes showed even more extensive changes. Notably, both JA and SA signaling pathways were drastically induced in L and $\mathrm{R}$ when infected with Aa (Figure 5a,b, Supplemental Table S3). 

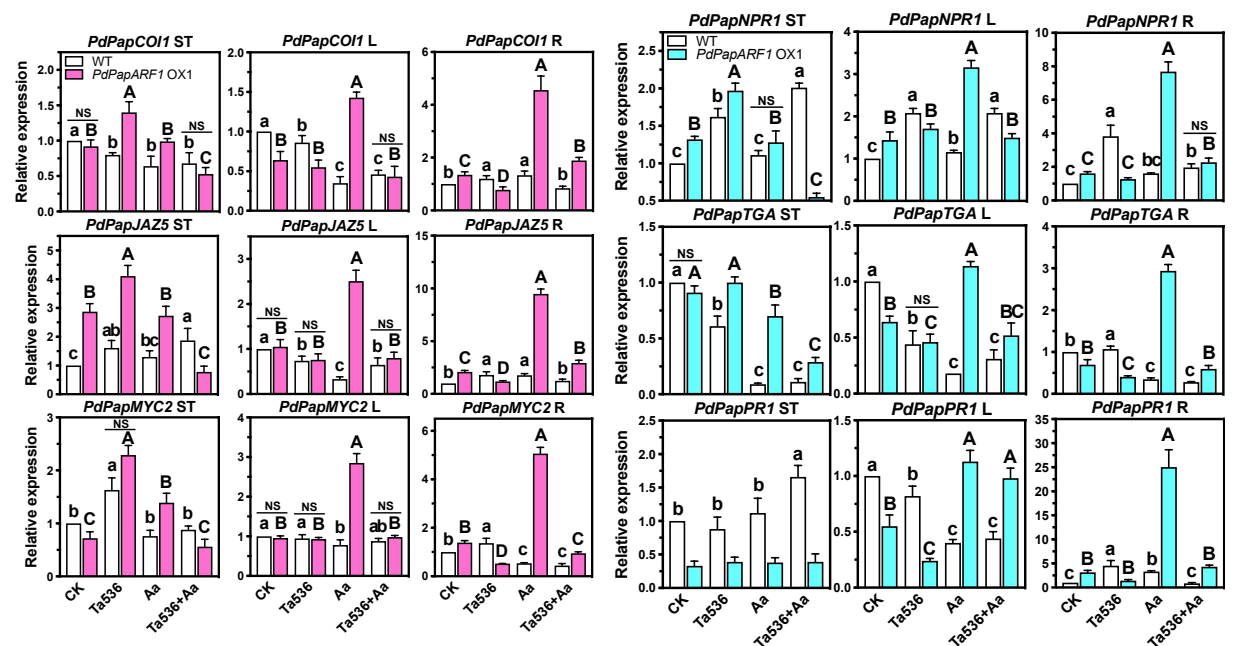

(a)

(b)
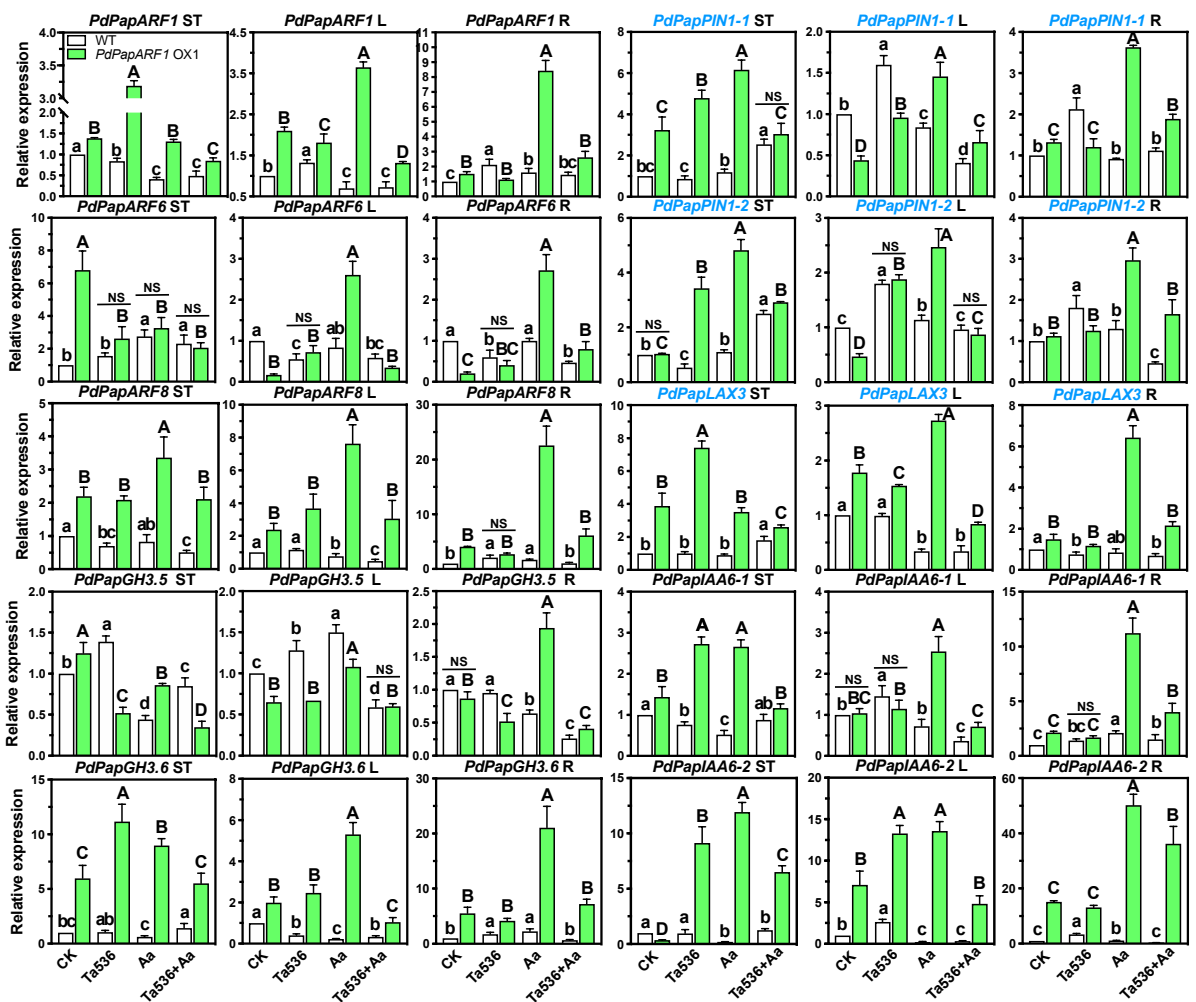

(c)

Figure 5. The expression of hormone-signaling-related genes of JA (a), SA (b), and IAA (c) in response to different fungal inoculations determined by q-RT-PCR. CK, uninoculated control. ST, shoot tip. L, inoculated leaf. R, root. Names of signaling-related genes are in black font, and names of transportation-related genes are in blue. Different capital letters represent significant differences among the OX plants treated with different inoculations; different lowercase letters represent significant differences among the WT plants treated with different inoculations; insignificant differences are not marked. NS represents that the difference between OX and WT plants undergone the same inoculation is not significant; in the results not marked with NS, significant difference exists between the OX and WT plants. All significances were at $p<0.05$. All experiments were performed with three biological replicates with each replicate pooled of three plants and three technical replicates. The replicates of OX plants were the clones of the line OX1. 
Furthermore, based on the phenotypes of OX1 plants, we examined the expression of poplar orthologs of genes that play important roles in adventitious root formation in Arabidopsis thaliana and poplar orthologs of auxin flux carriers including PIN1 and LAX3. AtARF6 and AtARF8 had been confirmed as positive regulators of adventitious root initiation [26]. They controlled the initiation of adventitious root depending on the auxin signal, which inhibited the COI1 pathway of JA signaling [26,27]. Meanwhile, AtARF6 and AtARF8 were the positive regulators of AtGH3.5 and $A t G H 3.6$, which could regulate each other through unidentified mechanisms and regulate JA homeostasis under the depression of AtIAA6, AtIAA9, and/or AtIAA17 [27,28]. Our results demonstrated a pronounced increase in PdPapARF8 expression and strong positive responses of PdPapARF1, PdPapARF6, and PdPapARF8 toward Aa inducing (Figure 5c, Supplemental Table S3). PdPapGH3.5 and PdPapGH3.6 showed antagonistic regulations in the OX1 plants, PdPapGH3.6 being drastically induced, and PdPapGH3.5 was suppressed in most cases we tested. The expression of the two poplar orthologs PdPapIAA6-1 and PdPapIAA6-2 were activated in the OX1 plants, both in the root and other compartments. The expression of the auxin efflux carriers PdPapPIN1-1, PdPapPIN1-2, and the auxin influx carrier PdPapLAX3 were all largely induced in ST in the OX1 plants under all the inoculations we performed. Their expression was also largely modified in $\mathrm{L}$ and $\mathrm{R}$ under some of our treatments, especially Aa inoculation. Young shoot organs are major sites on auxin production [29]. Our results suggest that the modification of auxin fluxes is a significant event during the response of poplar to Trichoderma inoculation, and PdPapARF1 is a key player in this event.

\section{Discussion}

Our results demonstrated that the overexpression of PdPapARF1 in poplar could improve plant growth in a non-Trichoderma-dependent manner and had even better promoting effects than Ta536 inoculation (Figure 2d, Supplemental Table S1). The IAA, JA, SA levels, and the expression of the key genes on the signaling pathways of these hormones were altered differentially in separate poplar compartments (ST, L, and R), which possibly reflected the underlying functional mechanism of PdPAPARF1.

The large increases of the IAA level and the expression of the auxin flux carrier genes, PdPapPIN1s and PdPapLAX3, in the young tissues (ST) reflected highly promoted auxin production and transportation in the PdPapARF1 OX1 plants compared to WT. IAA levels in L and R of the untreated OX1 plants were also higher than WT, suggesting completely different auxin homeostasis of the OX1 plants compared to WT. These, we believe, are the reasons for the promoted growth represented by plant height and leaf count of the OX1 plants. However, when inoculated with Ta536 or Aa, the IAA levels dropped in L and R of the OX1 plants and maintained at low levels in WT (Figure 4, Supplemental Table S2). Trichoderma spp. might produce additional IAA-related molecules in vitro [9-11]. A high concentration of auxin could inhibit the growth and development of root [30]. The decrease of the IAA level in R of the OX1 plants while subjected to Ta536 inducing might be a measure of poplar to achieve optimal auxin concentration for root growth.

Most curiously, comparing the responses of the expression of the auxin-related genes examined in this study in WT or OX1 plants, multiple genes were most induced by Ta536 in the WT plants but were most induced by Aa in the OX plants instead. Genes showing such patterns included PdPapARF1 itself, PdPapARF8, PdpapPIN1-1, PdPapPIN1-2, PdPapIAA6-2 in L and R, PdPapLAX3, PdPapIAA6-1 in L (Figure 5c, Supplemental Table S3). Our DAB staining assays and growth monitoring results of the WT and OX1 plants demonstrated that the OX1 plants took stronger and faster measures against the Aa invasion on-site and better secured the well-being of uninvaded plant compartments, thus promoted plant growth compared with WT (Figure 3). Hence, those genes induced by Ta536 or Aa in the opposite manners, as PdPapARF1 itself, might be crucial factors for achieving the beneficial effects of PdPapARF1 overexpression.

The roles as modifiers of plant hormone homeostasis, especially auxin homeostasis, of biofertilizers have been well established [31]. However, the underlying mechanisms are not yet elucidated. We believe 
that the modification of auxin production, distribution, fluxes, and signaling pathways in the OX1 plants compared with the WT plants were the major factors that led to the favorable growth traits of the OX1 poplars. Excessive activation of auxin-related pathways could suppress SA-dependent plant resistance but activates JA-dependent resistance in turn [32]. Pathogens can deliver cytosolic effectors to undermine the host plants' SA-dependent immunity [33]. In the WT poplars, the Ta536 inoculation or the duel inducing of Ta536+Aa either decreased SA or JA levels in both ST and L or only induced slight increases. The SA level in ST was even down-regulated significantly by Aa infection. However, in the OX1 plants, JA levels largely increased in ST under all treatments and in L under Ta536+Aa-inducing compared to WT. The SA-dependent and JA-dependent defense mechanisms in plants are mostly antagonistic [34]. However, in the OX1 plants, the JA and SA levels in R were both elevated, and the SA production in ST was not inhibited. The SA levels in L were lower than WT but increased under the Aa invasion (Figure 4, Supplemental Table S2). The SA pathways in plants mainly respond to biotrophic pathogens, while the JA pathways respond to necrotrophic pathogens [13]. A. alternata is a necrotrophic fungal pathogen, which may explain the drastic increase of the JA level in the infected leaves of the OX1 plants. The distinct hormone homeostasis profiles of OX1 plants and WT plants suggest a role of PdPapARF1 as a nexus for regulating the metabolism of major defense-related hormones, namely SA and JA.

Compared with the untreated WT plants, in the OX1 plants, the expression of JA and SA signaling-related genes was altered in the same manners as WT was by Ta536 inducing in all three examined compartments, except for a few cases, namely PdPapMYC2 in ST, PdPapJAZ5 in L and PdPapTGA in R. Such results demonstrated that the overexpression of PdPapARF1 served highly similar purposes as Ta536 inducing concerning the SA and JA signaling pathways, although the extent of regulation varied for each gene in each poplar compartment. Notably, when infected by Aa, in the OX1 plants, the expression of most of the examined genes was up-regulated drastically in some cases. Except that PdPapPR1 was down-regulated in ST. In contrast, in WT plants, some of these examined genes were down-regulated under Aa inducing, which was possibly due to the suppressing of plant immune responses by the pathogen [33]. Such results suggested distinct reacting mechanisms toward pathogen in the OX1 plants compared to WT. Combining our DAB staining results, the responses to pathogen in the OX1 plants might have been enhanced compared to WT. However, when Ta536 inducing was present, the examined JA and SA signaling-related genes showed varied regulation patterns in OX1 compared with WT. When Ta536 and Aa inducing were performed simultaneously, gene expression showed similar levels in OX1 and WT (Figure 5a,b, Supplemental Table S3). These results indicated that the effects of Ta536 inducing and PdPapARF1 overexpression might have both overlaps and antagonisms on the JA and SA signaling cascades in poplar, which requests further investigations. To conclude, our study demonstrated that PAPAPARF1 was a key player in poplar's response to Ta536, and its overexpression could benefit the poplar in similar or even better manners as did Ta536 inoculation.

\section{Materials and Methods}

\subsection{Plant, Fungal Material, and Growth}

The Populus davidiana $\times$ P. alba var. Pyramidalis plants were preserved by the Northeast Forestry University. Plants were subcultured on solid woody plant medium (WPM) for three weeks, then cut and cultured in liquid WPM for rooting for ten days for inoculation or transplanting into pot soil. Seedlings were cultured in a phytotron at $26{ }^{\circ} \mathrm{C}$ with a $16 / 8$-h light/dark cycle. The T. asperellum strains ACCC30536, ACCC32492, and ACCC31650 were purchased from the Center of Agricultural Culture Collection of China (ACCC). The A. alternata strain and the T. asperellum strain T4 was kindly provided by Dr. Zhi-Hua Liu of Shenyang Agricultural University. Fungal strains were cultured on the potato dextrose agar (PDA) medium at $26^{\circ} \mathrm{C}$ in dark for five days before being used as inoculums. Poplar 
plants in pot soil for monitoring growth were grown in a nursery at $26{ }^{\circ} \mathrm{C}$ with a $16 / 8$-h light/dark cycle. Three plants were used in each treatment group as three biological replicates.

\subsection{Cloning of PdPapARF1 Sequences and Production of Transgenic Poplars}

Total RNA and genomic DNA (gDNA) were extracted from young poplar leaves using TRIzol reagent (Invitrogen, Carlsbad, CA, USA) or the DNA extraction kit of Magen (Guangzhou, China). The extracted RNA was examined with electrophoresis and NanoDrop2000 (Thermo, DE, USA). The synthesis of cDNA was performed using the RT reagent Kit with gDNA Eraser (TaKaRa, Dalian, China). The amplification of PdPapARF1 sequences was performed using PrimeSTAR ${ }^{\circledR}$ Max (TaKaRa, Dalian, China). The sequencing of amplification products was performed by Tsingke, Beijing. Sequences of P. trichocarpa and A. thaliana were acquired from Phytozome 12.

The cds of PAPApARF1 was inserted into the pROKII expression vector at the SmaI site using the In-Fusion HD Cloning Kit (Clontech, CA, USA) to obtain the pCaMV35s::PdPapARF1 (OX) vector. Inverted repeat sequences of a 209-bp specific fragment of PdPapARF1 were amplified and used to construct the RNAi vector pFGC-PdPap-arf1 (RNAi) (the boxed sequence in Supplemental material 1). The designed RNAi target sequence of PdPapARF1 was inserted into the SwaI and SmaI sites of pFGC5941 vector as inverted repeat sequences to obtain the RNAi vector. The constructed vectors were then used to obtain transgenic poplar using Agrobacterium-mediated transformation and via regeneration in callus [35]. We examined the insertion of PdPapARF1 cds by amplifying a fragment from the gDNA using inter-extron primers to screen for overexpression plants. Plants with insertion showed both the cds amplification product and the gDNA product. A pair of primers was also used to examine the insertion of the fragment from the RNAi vector in the gDNA of the transformed plants. Meanwhile, qRT-PCR assays were performed to determine the expression of PdPapARF1 using the TransStar Top Green qPCR SuperMix (TransGen Biotech, Beijing, China) kit and the leaves as testing material. All procedures followed the manufacturers' instructions. Please see the supplemental material for all the primers used in this study (Supplemental Table S4).

\subsection{Inoculation of Plants}

For the data of Figure 1, plants were inoculated as described in our previous study [22,23]. For all the other data, plants were inoculated as below. The conidia of Ta536 were harvested through rinsing with $1 / 10$ strength liquid WPM and adjusted to $10^{3} \mathrm{cfu} / \mathrm{mL}$ concentration using a hemocytometer and microscope. The adjusted conidia suspension was then used as inoculum. For Ta536 inoculation, plants were cultured with the root immersed in the inoculum for $48 \mathrm{~h}$. For Aa inoculation (infection), the fifth and sixth leaves of the plant were punctured using a needle. Then, a 5-mm-diameter A. alternata mycelia disk was placed on the puncture wound, then the plant was cultured for $48 \mathrm{~h}$. Both procedures were performed on one plant as duel inoculation (Ta536+Aa). Identical treatments using clean 1/10 liquid WPM and PDA disks were performed as the mock inoculation to induce the control (CK) plants. All procedures were performed under sterile conditions.

\subsection{DAB Staining Assays, Determination of Hormone concentrations, qRT-PCR, and Statistic Analyses}

Accumulation of hydrogen peroxide $\left(\mathrm{H}_{2} \mathrm{O}_{2}\right)$ was detected using 3,3'- diaminobenzidine (DAB) staining assays, according to Hernandez et al. [36]. The two inoculated leaves (fifth, sixth) and two neighboring leaves (seventh, eighth) were stained for comparison. After the inoculation treatments (48 h), the 5-mm-length of shoot tip was harvested as ST. The inoculated leaves were harvested as L. All adventitious roots were harvested as $\mathrm{R}$. The hormone concentrations were examined as described in our previous study [37]. The same plant compartments ST, L, and R were used for qRT-PCR assays as described above. All the qRT-PCR assays were performed with three internal reference genes, PdPapACT1, PdPapEF1- $\alpha$, and PdPapUBQ, with the GenBank accession numbers KP973950, KP973951, and KP973952, respectively. Relative expression was calculated by $2^{-\Delta \Delta C t}$ using the mean expression of the three internal reference genes according to a previous publication [38]. Three biological replicates 
of WT plants and three replicates of OX1 plant clones were performed for all the qRT-PCR assays, each with three technical replicates. Statistical analyses were performed using SigmaPlot11.0 (Systat Software Inc., San Jose, CA, USA). Data are expressed as means \pm SD. One-way ANOVA and Student's t-test following Fisher's exact test were used to evaluate the statistical significance.

Supplementary Materials: The following are available online at http://www.mdpi.com/2223-7747/9/2/272/s1, Supplementary Figure S1: The phonotypes of PdPapARF1 RNAi poplar plants compared to a wild-type plant and an OX1 plant. Table S1: Data for Figure 2d. Table S2: Data for Figure 4. Table S3: Data for Figure 5. Table S4: All the primers used in this study, Supplementary material 1, the cds of PdPapARF1 showing the fragment used for constructing the RNAi vector.

Author Contributions: Conceptualization, R.-S.Z. and Y.-F.W.; methodology, Z.-H.Y., M.-M.L., and Y.-F.W.; software, X.-Y.H., Y.-F.W., and J.-J.D.; validation, Y.-F.W., R.-S.Z., X.-Y.H., and J.-J.D.; formal analysis, Y.-F.W., X.-Y.H., and J.-J.D.; investigation, Z.-H.Y. and M.-M.L.; resources, Z.-H.Y., M.-M.L., X.-Y.H., and J.-J.D.; data curation, Y.-F.W., R.-S.Z., Z.-H.Y., and M.-M.L.; writing-original draft preparation, Y.-F.W. and X.-Y.H.; writing-review and editing, Y.-F.W., X.-Y.H., J.-J.D., and R.-S.Z.; visualization, Y.-F.W. and X.-Y.H.; supervision, R.-S.Z. and Y.-F.W.; project administration, R.-S.Z. and Y.-F.W.; funding acquisition, R.-S.Z. All authors have read and agreed to the published version of the manuscript.

Funding: This research was funded by the National Natural Science Foundation of China (grant No. NSFC: 31370642) and the Joint Guidance (Lian-He-Yin-Dao) project of the Natural Science Foundation of Heilongjiang Province (grant No. LH2019C015).

Conflicts of Interest: The authors declare no conflict of interest.

\section{References}

1. Topolovec-Pintarić, S. Trichoderma: Invisible Partner for Visible Impact on Agriculture. In Trichoderma-The Most Widely Used Fungicide; IntechOpen: London, UK, 2019.

2. Chaverri, P.; Gazis, R.O.; Samuels, G.J. Trichoderma amazonicum, a new endophytic species on Hevea brasiliensis and H. guianensis from the Amazon basin. Mycologia 2011, 103, 139-151. [CrossRef]

3. Adnan, M.; Islam, W.; Shabbir, A.; Khan, K.A.; Ghramh, H.A.; Huang, Z.; Chen, H.Y.; Lu, G.-D. Plant defense against fungal pathogens by antagonistic fungi with Trichoderma in focus. Microb. Pathog. 2019, 129, 7-18. [CrossRef]

4. Harman, G.; Doni, F.; Khadka, R.B.; Uphoff, N. Endophytic strains of Trichoderma increase plants' photosynthetic capability. J. Appl. Microbiol. 2019, 1-18. [CrossRef]

5. Martinez-Medina, A.; Pozo, M.J.; Cammue, B.P.; Vos, C.M. Belowground defence strategies in plants: The plant-Trichoderma dialogue. In Belowground Defence Strategies in Plants; Springer: Berlin, Germany, 2016; pp. 301-327.

6. Patel, J.; Teli, B.; Bajpai, R.; Meher, J.; Rashid, M.; Mukherjee, A.; Yadav, S.K. Trichoderma-mediated biocontrol and growth promotion in plants: An endophytic approach. In Role of Plant Growth Promoting Microorganisms in Sustainable Agriculture and Nanotechnology; Elsevier: Amsterdam, The Netherlands, 2019; pp. 219-239.

7. Hermosa, R.; Viterbo, A.; Chet, I.; Monte, E. Plant-beneficial effects of Trichoderma and of its genes. Microbiology 2012, 158, 17-25. [CrossRef]

8. Stewart, A.; Hill, R. Applications of Trichoderma in plant growth promotion. In Biotechnology and Biology of Trichoderma; Elsevier: Amsterdam, The Netherlands, 2014; pp. 415-428.

9. Contreras-Cornejo, H.A.; Macías-Rodríguez, L.; Cortés-Penagos, C.; López-Bucio, J. Trichoderma virens, a plant beneficial fungus, enhances biomass production and promotes lateral root growth through an auxin-dependent mechanism in Arabidopsis. Plant Physiol. 2009, 149, 1579-1592. [CrossRef] [PubMed]

10. Gravel, V.; Antoun, H.; Tweddell, R.J. Growth stimulation and fruit yield improvement of greenhouse tomato plants by inoculation with Pseudomonas putida or Trichoderma atroviride: Possible role of indole acetic acid (IAA). Soil Biol. Biochem. 2007, 39, 1968-1977. [CrossRef]

11. Zhang, S.; Gan, Y.; Xu, B. Mechanisms of the IAA and ACC-deaminase producing strain of Trichoderma longibrachiatum T6 in enhancing wheat seedling tolerance to NaCl stress. BMC Plant Biol. 2019, 19, 22. [CrossRef] [PubMed]

12. Yang, J.; Duan, G.; Li, C.; Liu, L.; Han, G.; Zhang, Y.; Wang, C. The Crosstalks Between Jasmonic Acid and Other Plant Hormone Signaling Highlight the Involvement of Jasmonic Acid as a Core Component in Plant Response to Biotic and Abiotic Stresses. Front. Plant Sci. 2019, 10, 1349. [CrossRef] [PubMed] 
13. Pieterse, C.M.; Van der Does, D.; Zamioudis, C.; Leon-Reyes, A.; Van Wees, S.C. Hormonal modulation of plant immunity. Annu. Rev. Cell Dev. Biol. 2012, 28, 489-521. [CrossRef]

14. Zemlyanskaya, E.V.; Omelyanchuk, N.A.; Ubogoeva, E.V.; Mironova, V.V. Deciphering auxin-ethylene crosstalk at a systems level. Int. J. Mol. Sci. 2018, 19, 4060. [CrossRef] [PubMed]

15. Singh, A.; Shukla, N.; Kabadwal, B.; Tewari, A.; Kumar, J. Review on Plant-Trichoderma-Pathogen Interaction. Int. J. Curr. Microbiol. Appl. Sci. 2018, 7, 2382-2397. [CrossRef]

16. Engelberth, J.; Koch, T.; Schüler, G.; Bachmann, N.; Rechtenbach, J.; Boland, W. Ion channel-forming alamethicin is a potent elicitor of volatile biosynthesis and tendril coiling. Cross talk between jasmonate and salicylate signaling in lima bean. Plant Physiol. 2001, 125, 369-377. [CrossRef]

17. Tucci, M.; Ruocco, M.; De Masi, L.; De Palma, M.; Lorito, M. The beneficial effect of Trichoderma spp. on tomato is modulated by the plant genotype. Mol. Plant Pathol. 2011, 12, 341-354. [CrossRef]

18. Yoshioka, Y.; Ichikawa, H.; Naznin, H.A.; Kogure, A.; Hyakumachi, M. Systemic resistance induced in Arabidopsis thaliana by Trichoderma asperellum SKT-1, a microbial pesticide of seedborne diseases of rice. Pest Manag. Sci. 2012, 68, 60-66. [CrossRef]

19. Züst, T.; Agrawal, A.A. Trade-offs between plant growth and defense against insect herbivory: An emerging mechanistic synthesis. Annu. Rev. Plant Biol. 2017, 68, 513-534. [CrossRef]

20. Kunkel, B.N.; Harper, C.P. The roles of auxin during interactions between bacterial plant pathogens and their hosts. J. Exp. Bot. 2017, 69, 245-254. [CrossRef]

21. Li, S.-B.; Xie, Z.-Z.; Hu, C.-G.; Zhang, J.-Z. A review of auxin response factors (ARFs) in plants. Front. Plant Sci. 2016, 7, 47. [CrossRef]

22. Liu, Z.-Y.; Jiang, C.-Y.; Zhai, T.-T.; Chang, Y.; Yao, Z.-H.; Zhang, R.-S. Effects of Trichoderma asperellum combined application on growth and photosynthesis characteristics of Populus davidiana $\times$ P. alba var. pyramidlis. Bull. Bot. Res. 2018, 38, 64-74. [CrossRef]

23. Jiang, C.-Y.; Zhu, G.-D.; Yao, Z.-H.; Yang, X.-T.; Liu, Z.-H.; Zhang, R.-S. Effects of three Trichoderma asperellum strains on the growth and Photosynthetic characteristics of tissue-cultured Populus davidiana $X$ P. alba var. Pyramidalis seedlings. Pratacult. Sci. 2016, 33, 1189-1199.

24. Apostol, I.; Heinstein, P.F.; Low, P.S. Rapid stimulation of an oxidative burst during elicitation of cultured plant cells: Role in defense and signal transduction. Plant Physiol. 1989, 90, 109-116. [CrossRef]

25. Li, Y.; Feng, Y.; Lü, Q.; Yan, D.; Liu, Z.; Zhang, X. Comparative Proteomic Analysis of Plant-Pathogen Interactions in Resistant and Susceptible Poplar Ecotypes Infected with Botryosphaeria dothidea. Phytopathology 2019, 109, 2009-2021. [CrossRef]

26. Gutierrez, L.; Bussell, J.D.; Păcurar, D.I.; Schwambach, J.; Păcurar, M.; Bellini, C. Phenotypic plasticity of adventitious rooting in Arabidopsis is controlled by complex regulation of AUXIN RESPONSE FACTOR transcripts and microRNA abundance. Plant Cell 2009, 21, 3119-3132. [CrossRef]

27. Gutierrez, L.; Mongelard, G.; Floková, K.; Păcurar, D.I.; Novák, O.; Staswick, P.; Kowalczyk, M.; Păcurar, M.; Demailly, H.; Geiss, G. Auxin controls Arabidopsis adventitious root initiation by regulating jasmonic acid homeostasis. Plant Cell 2012, 24, 2515-2527. [CrossRef]

28. Lakehal, A.; Chaabouni, S.; Cavel, E.; Le Hir, R.; Ranjan, A.; Raneshan, Z.; Novák, O.; Păcurar, D.I.; Perrone, I.; Jobert, F. A Molecular Framework for the Control of Adventitious Rooting by TIR1/AFB2-Aux/IAA-Dependent Auxin Signaling in Arabidopsis. Mol. Plant 2019, 12, 1499-1514. [CrossRef]

29. Aloni, R.; Aloni, E.; Langhans, M.; Ullrich, C. Role of cytokinin and auxin in shaping root architecture: Regulating vascular differentiation, lateral root initiation, root apical dominance and root gravitropism. Ann. Bot. 2006, 97, 883-893. [CrossRef]

30. Evans, M.L.; Ishikawa, H.; Estelle, M.A. Responses of Arabidopsis roots to auxin studied with high temporal resolution: Comparison of wild type and auxin-response mutants. Planta 1994, 194, 215-222. [CrossRef]

31. Tsukanova, K.; Meyer, J.; Bibikova, T. Effect of plant growth-promoting Rhizobacteria on plant hormone homeostasis. S. Afr. J. Bot. 2017, 113, 91-102. [CrossRef]

32. Naseem, M.; Kaltdorf, M.; Dandekar, T. The nexus between growth and defence signalling: Auxin and cytokinin modulate plant immune response pathways. J. Exp. Bot. 2015, 66, 4885-4896. [CrossRef]

33. Asai, S.; Shirasu, K. Plant cells under siege: Plant immune system versus pathogen effectors. Curr. Opin.Plant Biol. 2015, 28, 1-8. [CrossRef]

34. Caarls, L.; Pieterse, C.M.; Van Wees, S. How salicylic acid takes transcriptional control over jasmonic acid signaling. Front. Plant Sci. 2015, 6, 170. [CrossRef] 
35. Deng, W.; Luo, K.; Li, Z.; Yang, Y. A novel method for induction of plant regeneration via somatic embryogenesis. Plant Sci. 2009, 177, 43-48. [CrossRef]

36. Hernández, J.A.; Ferrer, M.A.; Jiménez, A.; Barceló, A.R.; Sevilla, F. Antioxidant systems and $\mathrm{O}^{2-} / \mathrm{H}_{2} \mathrm{O}_{2}$ production in the apoplast of pea leaves. Its relation with salt-induced necrotic lesions in minor veins. Plant Physiol. 2001, 127, 817-831. [CrossRef]

37. Zhai, T.; Wang, Y.; Baloch, A.M.; Baloch, A.W.; Liu, Z.; Jiang, C.; Zhang, R. Trichoderma aspereullm ACCC30536 inoculation differently regulates the time-course expression of five indole-3-acetic acid amido synthetase genes and the levels of IAA, SA and JA in Populus davidiana $\times$ P. alba var. Pyramidalis. Pak. J. Bot. 2019, 51, 689-697. [CrossRef]

38. Livak, K.J.; Schmittgen, T.D. Analysis of relative gene expression data using real-time quantitative PCR and the 2- $\Delta \Delta$ CT method. Methods 2001, 25, 402-408. [CrossRef]

(C) 2020 by the authors. Licensee MDPI, Basel, Switzerland. This article is an open access article distributed under the terms and conditions of the Creative Commons Attribution (CC BY) license (http://creativecommons.org/licenses/by/4.0/). 